\title{
GENERALISATION OF AN INTEGRAL \\ DUE TO HARDY
}

\author{
by FOUAD M. RAGAB
}

(Received 30th April, 1951)

§. Introductory. The integral

$$
\int_{0}^{\infty} K_{n}(t) K_{n}\left(\frac{b}{t}\right) d t=\pi K_{2 n}(2 \sqrt{ } b)
$$

where $b>0$, was given by Hardy (1). It was proved by applying Mellin's inversion formula. An alternative proof, based on the differential equation

$$
x^{2} y^{\prime \prime}+x y^{\prime}-\left(x^{2}+n^{2}\right) y=0
$$

satisfied by $K_{n}(x)$, has been given by the author (2).

In $\S 2$, a generalisation of this formula, namely

$$
\prod_{s=1}^{p-1} \int_{0}^{\infty} K_{n}\left(t_{s}\right) t_{s}^{2 s / p-1} d t_{s} K_{n}\left(\frac{b}{t_{1} t_{2} \ldots t_{p-1}}\right)=\pi^{p-1} K_{p n}\left(p b^{1 / p}\right)
$$

where $b>0, p=2,3,4, \ldots$, will be established.

The following formulae will be required in the proof :

$$
\int_{0}^{\infty} \lambda^{m-1} K_{n}(\lambda) d \lambda=2^{m-2} \Gamma\left(\frac{m+n}{2}\right) \Gamma\left(\frac{m-n}{2}\right),
$$

where $R(m \pm n)>0(3)$; and

$$
\Gamma(z) \Gamma\left(z+\frac{1}{m}\right) \ldots \Gamma\left(z+\frac{m-1}{m}\right)=(2 \pi)^{\frac{1}{m} m-\frac{1}{2}} m^{\frac{1}{2}-m z} \Gamma(m z),
$$

where $m$ is a positive integer (4).

In $\$ 3$ a similar formula, involving Bessel Functions of the First Kind, will be obtained.

§2. Proof of the Formula. If the L.H.S. of (3) is denoted by $F(b)$, then

and

$$
F^{\prime}(b)=\prod_{s=1}^{p-1} \int_{0}^{\infty} K_{n}\left(t_{s}\right) t_{s}^{2 s / p-1} d t_{s} K_{n}^{\prime}\left(\frac{b}{t_{1} t_{2} \ldots t_{p-1}}\right) \frac{1}{t_{1} t_{2} \ldots t_{p-1}}
$$

Then, from (2),

$$
F^{\prime \prime}(b)=\prod_{s=1}^{p-1} \int_{0}^{\infty} K_{n}\left(t_{s}\right) t_{s}^{2 s / p-1} d t_{s} K_{n}^{\prime \prime}\left(\frac{b}{t_{1} t_{2} \ldots t_{p-1}}\right) \frac{1}{\left(t_{1} t_{2} \ldots t_{p-1}\right)^{2}}
$$

$$
\begin{aligned}
b^{2} F^{\prime \prime}(b)=\prod_{s=1}^{p-1} \int_{0}^{\infty} K_{n}\left(t_{s}\right) t_{s}^{2 s / p-1} d t_{s} & \\
\times & {\left[-\frac{b}{t_{1} t_{2} \ldots t_{p-1}} K_{n}^{\prime}\left(\frac{b}{t_{1} t_{2} \ldots t_{p-1}}\right)+\left\{\left(\frac{b}{t_{1} t_{2} \ldots t_{p-1}}\right)^{2}+n^{2}\right\} K_{n}\left(\frac{b}{t_{1} t_{2} \ldots t_{p-1}}\right)\right] } \\
= & -b F^{\prime}(b)+n^{2} F(b)+L,
\end{aligned}
$$

where

$$
L \equiv b^{2} \prod_{s=1}^{p-1} \int_{0}^{\infty} K_{n}\left(t_{s}\right) t_{s}^{2 s / p-3} d t_{s} K_{n}\left(\frac{b}{t_{1} t_{2} \ldots t_{p-1}}\right) .
$$


In this multiple integral change the order of integration so that the first integral becomes the last and replace $t_{1}$ by $b /\left(\lambda t_{2} t_{3} \ldots t_{p-1}\right)$, where $\lambda$ is the new variable ; then

$$
\begin{aligned}
L= & b^{2} \prod_{s=2}^{p-1} \int_{0}^{\infty} K_{n}\left(t_{s}\right) t_{s}^{2 s / p-3} d t_{s} \\
& \times \int_{0}^{\infty} K_{n}(\lambda)\left(\frac{b}{\lambda t_{2} \ldots t_{p-1}}\right)^{2 / p-3} K_{n}\left(\frac{b}{\lambda t_{2} \ldots t_{p-1}}\right) \frac{b d \lambda}{\lambda^{2} t_{2} \ldots t_{p-1}} \\
=b^{2 / p} & \prod_{s=2}^{p-1} \int_{0}^{\infty} K_{n}\left(t_{s}\right) t_{s}{ }^{2(s-1) / p-1} d t_{s} \\
& \times \int_{0}^{\infty} K_{n}(\lambda) \lambda^{2(p-1) / p-1} K_{n}\left(\frac{b}{\lambda t_{2} t_{3} \ldots t_{p-1}}\right) d \lambda .
\end{aligned}
$$

Here write $t_{s-1}$ for $t_{s}$ and $t_{p-1}$ for $\lambda$ : then

so that

$$
L=b^{2 / p} F(b)
$$

$$
b^{2} F^{\prime \prime}(b)+b F^{\prime}(b)-\left(b^{2 / p}+n^{2}\right) F(b)=0 .
$$

Now, in (2) put $b=(x / p)^{p}$, and it becomes

and therefore

$$
b^{2} \frac{d^{2} y}{d b^{2}}+b \frac{d y}{d b}-\left(b^{2 / p}+n^{2} / p^{2}\right) y=0:
$$

$$
F(b)=A K_{p n}\left(p b^{1 / p}\right)+B I_{p n}\left(p b^{1 / p}\right) .
$$

Here let $b \rightarrow \infty$ and it is seen that $B$ must be zero. (For the purpose of the proof it may be assumed for the time being that $n \geqq 0$.)

In order to determine $A$ the equation may be put in the form

$$
\begin{gathered}
\prod_{s=1}^{p-1} \int_{0}^{\infty} K_{n}\left(t_{s}\right) t_{s}^{2 s / p-1} d t_{s} \frac{\pi}{2 \sin n \pi}\left\{I_{-n}\left(\frac{b}{t_{1} \ldots t_{p-1}}\right)-I_{n}\left(\frac{b}{t_{1} \ldots t_{p-1}}\right)\right\} \\
=A \frac{\pi}{2 \sin (p n \pi)}\left\{I_{-p n}\left(p b^{1 / p}\right)-I_{p n}\left(p b^{1 / p}\right)\right\} .
\end{gathered}
$$

Now multiply by $b^{n}$ and let $b \rightarrow 0$; then or, from (4),

$$
\prod_{8=1}^{p-1} \int_{0}^{\infty} K_{n}\left(t_{s}\right) t_{s}^{2 s / p-1} d t_{s} \frac{2^{n} \pi}{2 \sin n \pi} \frac{\left(t_{1} t_{2} \ldots t_{p-1}\right)^{n}}{\Gamma(1-n)}=A \frac{\pi(2 / p)^{p n}}{2 \sin (p n \pi)} \frac{1}{\Gamma(1-p n)},
$$

$$
\prod_{8=1}^{p-1} 2^{n+2 s / p-2} \Gamma(n+s / p) \Gamma(s / p) 2^{n-1} \Gamma(n)=A 2^{p n-1} p^{-p n} \Gamma(p n) .
$$

Hence, from (5) and from (5) with $1 / m$ in place of $z$,

$$
2^{(p-1) n-(p-1)+n-1}(2 \pi)^{\frac{1}{2} p-\frac{1}{2}} p^{\frac{1}{2}-p n} \Gamma(p n)(2 \pi)^{\frac{1}{1} p-\frac{1}{2}} p^{-\frac{1}{2}}=A 2^{p n-1} p^{-p n} \Gamma(p n) .
$$

Therefore,

$$
A=\pi^{p-1} \text {. }
$$

Thus formula (3) has been established.

§ 3. A Multiple Integral involving Bessel Functions of the First Kind. The formula to be proved is

$$
\prod_{s=1}^{p-1} \int_{0}^{\infty} J_{n}\left(t_{s}\right) t_{s}^{2 s / p-1} d t_{s} J_{n}\left(\frac{b}{t_{1} t_{2} \ldots t_{p-1}}\right)=J_{p n}\left(p b^{1 / p}\right)
$$

where $b>0, R(n)>\frac{1}{2}-\frac{2}{p}$. For the particular case, when $p=2$,

$$
\int_{0}^{\infty} J_{n}(t) J_{n}\left(\frac{b}{t}\right) d t=J_{2 n}(2 \sqrt{ } b)
$$

where $b>0, R(n)>-\frac{1}{2}$, see Watson's Bessel Functions (5). 
The proof depends on the differential equation

satisfied by $J_{n}(x)$ and $J_{-n}(x)$.

$$
x^{2} y^{\prime \prime}+x y^{\prime}+\left(x^{2}-n^{2}\right) y=0,
$$

The formula

$$
\int_{0}^{\infty} J_{n}(\lambda) \lambda^{m-1} d \lambda=2^{m-1} \Gamma\left(\frac{m+n}{2}\right) / \Gamma\left(1+\frac{n-m}{2}\right)
$$

where $R(n+m)>0, R(m)<\frac{3}{2}$, is required (6).

Denoting the L.H.S. of $(6)$ by $\phi(b)$, we have, as in $\S 2$, if $R(n)>\frac{3}{2}-\frac{2}{p}$,

where

$$
b^{2} \phi^{\prime \prime}(b)=-b \phi^{\prime}(b)+n^{2} \phi(b)-L,
$$

$$
L=b^{2} \prod_{s=1}^{p-1} \int_{0}^{\infty} J_{n}\left(t_{s}\right) t_{s}^{2 s / p-3} d t_{s} J_{n}\left(\frac{b}{t_{1} t_{2} \ldots t_{p-1}}\right) .
$$

On proceeding as in $\S 2$, it is found that

so that

$$
L=b^{2 / p} \phi(b),
$$

$$
b^{2} \phi^{\prime \prime}(b)+b \phi^{\prime}(b)+\left(b^{2 / p}-n^{2}\right) \phi(b)=0 .
$$

Now, in (8), put $b=(x / p)^{p}$ and it becomes

Therefore

$$
b^{2} \frac{d^{2} y}{d b^{2}}+b \frac{d y}{d b}+\left(b^{2 / p}-\frac{n^{2}}{p^{2}}\right) y=0 .
$$

$$
\phi(b)=A J_{p n}\left(p b^{1^{\prime p}}\right)+B J_{-p n}\left(p b^{1 / p}\right) .
$$

Here multiply by $b^{n}$ and let $b \rightarrow 0$; then clearly $B$ must be zero.

Again, to determine $A$, multiply by $b^{-n}$ and let $b \rightarrow 0$; then

$$
\prod_{s=1}^{p-1} \int_{0}^{\infty} J_{n}\left(t_{s}\right) t_{s}^{2 s / p-n-1} d t_{s} \frac{1}{2^{n} \Gamma(n+1)}=A \frac{p^{p n}}{2^{p n} \Gamma(p n+1)} .
$$

But, from (9), the L.Fr.S. is equal to

$$
\prod_{8=1}^{p-1} 2^{2 s / p-n-1} \Gamma\left(\frac{s}{p}\right) / \Gamma\left(n+\frac{p-s}{p}\right) \times \frac{1}{2^{n} \Gamma(n+1)}=\frac{2^{-n(p-1)}(2 \pi)^{\frac{1}{p} p-\frac{1}{2}} p^{-\frac{1}{3}}}{2^{n} n(2 \pi)^{\frac{1}{2} p-\frac{1}{2}} p^{\frac{1}{b^{-p}} \Gamma(p n)}} .
$$

Hence $A=1$, so that (6) has been proved. By applying analytical continuation the restriction $R(n)>\frac{3}{2}-\frac{2}{p}$ can be altered to $R(n)>\frac{1}{2}-\frac{2}{p}$.

\section{REFERENCES}

(1) Hardy, G. H., Mess. of Maths., 56, 190 (1927).

(2) Ragab, F. M., Proc. Glasg. Math. Ass., 1, 72 (1952).

(3) Gray, Mathews and MacRobert, Bessel Functions, p. 66.

(4) MacRobert, T. M., Complex Variable, p. 154.

(5) Watson, G. N., Bessel Functions, p. 437.

(6) MacRobert, T. M., Complex Variable (3rd ed.), p. 372.

\section{UNIVERSITy of GLasqow}

